\title{
46. ORIGINS OF METEORITIC MATERIAL
}

\author{
(Survey Paper)
}

\section{F. L. WHIPPLE,}

(Smithsonian Astrophysical Observatory,; and Harvard College Observatory, Cambridge, Mass., U.S.A.)

\section{A B STRACT}

The basic material of this discussion is being published under the title 'On Maintaining the Meteoritic Complex'.* The assumed meteoritic influx on the Earth is derived from measurements of penetration of space vehicles, radio and photographic meteors, meteorite falls, Apollo asteroids, lunar craters, and comets (see Figure 1). I assume that the much higher impact rates from acoustic measures of dust and from collections do not measure the true influx rate. The total flux is some

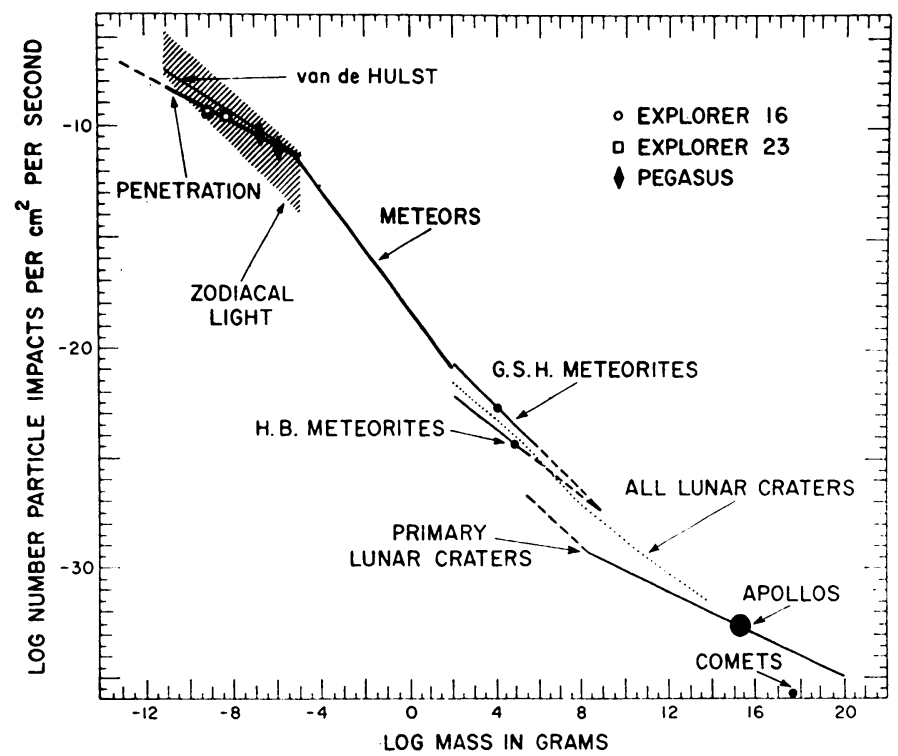

FIG. 1. Cumulative impact rates of meteoritic material on a sphere near the Earth's orbit.

$2 \times 10^{-16} \mathrm{~g} \mathrm{~cm}^{-2} \mathrm{sec}^{-1}$ on the surface of a corresponding non-gravitating sphere. I take the equivalent space density of some $2 \times 10^{-22} \mathrm{gm} \mathrm{cm}^{-3}$ as applicable over a volume of some $3.5 \mathrm{AU}$ radius about the Sun and $i<20^{\circ}$ of the ecliptic, giving a total mass of $4.5 \times 10^{19} \mathrm{gm}$ for particles of mass $<10^{2} \mathrm{gm}$. For Earth-crossing orbits the total mass is some $1.3 \times 10^{19} \mathrm{gm}$. I adopt $2.5 \times 10^{19} \mathrm{gm}$ for the total mass.

* Presented at the Conference on Zodiacal Light and the Interplanetary Medium, Honolulu, Hawaii, Jan.-Feb., 1967. 
All known dissipating or destructive factors are included in determining the 'ecology' of the meteoritic material. Direct light pressure quickly eliminates particles of the order of $1 \mu$ or less in dimension while the solar wind drives away all gases. The Poynting-Robertson effect ( $P R$-effect) is effectively increased by some $22 \%$ because of the pseudo $P R$-effect of the solar wind. Direct sublimation of earthy solids is significant only near the Sun while sputtering losses produced by the solar wind are real but effectively small $(\sim 10 \%)$ compared to spiralling rates by the $P R$-effect. Magnetic-field, charge and rotation effects may quite possibly be significant but are not yet subject to precise calculations.

Space erosion has been demonstrated for stony meteorites and cometary meteoroids (see Table 1). A totally destructive collision is assumed to occur when a particle of mass $m$ is struck by another with mass $\geqslant m / 3200$. Gravitational elimination effects for the Earth and Venus (time $\sim 10^{8} \mathrm{yr}$ ), Mars (time $\sim 6 \times 10^{9} \mathrm{yr}$ ) and Jupiter (time $\sim 10^{6} \mathrm{yr}$ ) are, following Öpik, assumed to be negligible for the small Zodiacal Cloud particles, compared to the dissipative effects included above.

\section{Table 1}

\section{Erosion in space}

$\begin{array}{lccc} & \text { Irons } & \text { Stones } & \text { Cometary meteors } \\ \text { Exposure age }(\mathrm{yr}) & \sim 5 \times 10^{8} & <5 \times 10^{7} & \sim 10^{4} \mathrm{a} \\ \text { Etching rate }\left(\mathrm{cm} \mathrm{yr}^{-1}\right) & 5 \times 10^{-8} & 5 \times 10^{-7} & 2.3 / \rho \times 10^{-5} \mathrm{~b} \\ \text { Etching rate }\left(\mathrm{g} \mathrm{cm}^{-2} \mathrm{sec}^{-1}\right) & 1.3 \times 10^{-14} & 5 \times 10^{-14} & 7 \times 10^{-13} \\ \text { Impact rate }\left(\mathrm{g} \mathrm{cm}^{-2} \mathrm{sec}^{-1}\right) & 6 \times 10^{-17} & 5 \times 10^{-17} & 20 \times 10^{-17}\end{array}$

a Average lifetime.

b $\rho=$ density.

Table 2

\section{Distribution of masses and lifetimes}

\begin{tabular}{|c|c|c|c|c|c|c|}
\hline \multirow[b]{2}{*}{$\begin{array}{c}\text { Particle } \\
\text { mass (g) } \\
\log m\end{array}$} & \multirow[b]{2}{*}{$\begin{array}{c}\text { Particle } \\
\text { radius }(\mathrm{cm}) \\
\log s\end{array}$} & \multirow{2}{*}{$\begin{array}{c}\text { Percent } \\
\text { mass } \\
10^{-0 \cdot 5} \mathrm{~m} \text { to } \\
10^{+0 \cdot 5} \mathrm{~m}\end{array}$} & \multicolumn{3}{|c|}{ Lifetimes (yr) } & \multirow[b]{2}{*}{$\begin{array}{l}\text { Mean } \\
\log \tau\end{array}$} \\
\hline & & & $\begin{array}{c}P R \\
\log \tau_{\mathrm{PR}}\end{array}$ & $\begin{array}{c}\text { Erosion } \\
\log \tau_{\mathrm{e}}\end{array}$ & $\begin{array}{c}\text { Collision } \\
\text { mean } \\
\log \tau_{\mathrm{e}}\end{array}$ & \\
\hline$-12 \cdot 0$ & $-4 \cdot 21$ & 0.02 & $3.06 ?$ & $6 \cdot 70$ & 5.92 & $3.06 ?$ \\
\hline-11.0 & -3.87 & 0.04 & $3 \cdot 40 ?$ & 6.54 & $5 \cdot 84$ & 3.40 ? \\
\hline$-10 \cdot 0$ & -3.54 & $0 \cdot 14$ & 3.73 & 6.39 & $5 \cdot 76$ & 3.73 \\
\hline-9.0 & $-3 \cdot 21$ & 0.44 & 4.06 & $6 \cdot 23$ & $5 \cdot 65$ & 4.05 \\
\hline-8.0 & -2.87 & 1.4 & 4.40 & 6.07 & 5.53 & 4.37 \\
\hline-7.0 & -2.54 & $4 \cdot 2$ & 4.73 & 5.92 & 5.41 & 4.66 \\
\hline-6.0 & -2.21 & 12.9 & 5.06 & $5 \cdot 76$ & $5 \cdot 28$ & 4.88 \\
\hline-5.0 & -1.76 & 40.9 & 5.51 & $5 \cdot 61$ & $5 \cdot 13$ & 5.00 \\
\hline-4.0 & -1.42 & $21 \cdot 5$ & 5.85 & 5.45 & 4.96 & 4.91 \\
\hline-3.0 & -1.09 & $10 \cdot 0$ & $6 \cdot 18$ & 5.29 & $4 \cdot 77$ & 4.75 \\
\hline-2.0 & -0.76 & $4 \cdot 6$ & $6 \cdot 51$ & $5 \cdot 14$ & 4.40 & 4.40 \\
\hline-1.0 & -0.42 & $2 \cdot 2$ & $6 \cdot 85$ & 4.97 & 5.02 & 4.79 \\
\hline 0.0 & -0.09 & 1.0 & $7 \cdot 18$ & $5 \cdot 17$ & 5.69 & $5 \cdot 10$ \\
\hline+1.0 & +0.24 & 0.5 & 7.51 & 5.45 & $6 \cdot 36$ & 5.42 \\
\hline+2.0 & +0.58 & 0.2 & $7 \cdot 85$ & $5 \cdot 76$ & 7.02 & 5.75 \\
\hline$>2.0$ & +0.58 & 0.2 & & & & \\
\hline
\end{tabular}

Weighted mean life of all particles, $T-8 \times 10^{4} \mathrm{yr}$. 
The mean lifetimes for small particles of mass $m$ are calculated crudely and presented in Table 2 along with the assumed distribution function in mass. The corrected (factor $1 / 1 \cdot 3$ ) $P R$-lifetime is indicated by $\tau_{\mathrm{PR}}$, erosion by particles of mass $<m / 3200$ by $\tau_{\mathrm{e}}$, collisional destruction by particles of mass $\geqslant m / 3200$ by $\tau_{c}$, and the mean lifetime including all these effects by $\tau$.

The mean lifetime is much less dependent on mass than might be expected. Averaging all the particles according to mass distribution and $\tau$, the weighted mean lifetime for all material of $m<10^{2} \mathrm{gm}$ comes out $8 \times 10^{4} \mathrm{yr}$. Combining this mean lifetime with a total mass of $2.5 \times 10^{19} \mathrm{gm}$, the average total-input rate to maintain the Zodiacal Cloud in quasi-equilibrium becomes some 10 tons sec $\mathrm{se}^{-1}$. Note that this input rate may be considerably overestimated because I have not included the contributions to the cloud by broken fragments.

It appears quite possible that comets can supply the needed 10 tons $\mathrm{sec}^{-1}$ injected into orbits totally within that of Jupiter. Fragile carbonaceous chondrites and 'half-baked asteroids' (see Appendix), however, may compete with comets in contributing to the fireballs and possibly to the visual meteors. The stony meteorites may be maintained by collisional spallation from Earthcrossing asteroids induced by smaller bodies, the Apollo asteroids being derived from the asteroid belt by the gravitational effects of Mars. The data and theory are not yet adequate to provide a definitive solution to the problem of asteroidal vs. cometary origin for the Apollo asteroids.

One asks whether McCrosky's fireballs might have originated directly from comets, not primarily from Apollo asteroids. Jupiter crossings $\left(\tau \sim 10^{6} \mathrm{yr}\right)$ might have eliminated most of the orbits with aphelia beyond Jupiter's. A rough calculation failed by a factor of 3-5 in accounting for the reduction in numbers of orbits with large aphelion distances among McCrosky's fireballs as compared to the number among photographic meteors. This leaves the question essentially unsolved but also allows the possibility that a considerable fraction of McCrosky's fireballs may be of direct cometary origin, in typical short-period comet orbits, rather than Apollo-asteroid fragments.

\section{Appendix: Pre-Type I Carbonaceous Chondrites?}

Is there in space a large quantity of meteoritic material of asteroidal origin that is more 'primitive', or less metamorphosed, in character, and more friable than the Type I carbonaceous chondrites? Such material could arise from the outer surfaces of larger asteroids in which the Type I carbonaceous chondrites were formed somewhat below the surface. It could also arise from the central regions of what we might call 'half-baked' asteroids, those of small dimensions that may not have been entirely melted in the early stages of solar-system evolution, and may not have been entirely eliminated to date.

Observationally, such material would be difficult to identify on Earth because of its fragility in atmospheric passage. Also, it could easily be confused with cometary material that might be roughly similar in density, friability, and even composition. In preliminary results from the Prairie Network fireball observing program, McCrosky (in the present volume, p. 265) reports that a surprisingly large fraction of extremely bright fireballs disappear at high altitudes and appear to be very friable. Is it possible that some or many of these objects may be not cometary but asteroidal in origin?

Could the great Tunguska fireball of 1908 have been a low-density $\left(\sim 1 \mathrm{gm} / \mathrm{cm}^{3}\right)$ pre-Type I carbonaceous chondrite rather than a small comet nucleus as suggested by Fesenkov (1961)?

The problem is confused theoretically on several counts. For example, Öpik (1966) 
now suggests seriously that all meteorites come from comets. Other theoretical questions concern details of asteroidal as well as cometary formation, particularly the initial thermal circumstances and the amount of radioactivity available at the time of formation for heating and melting. Could smaller asteroids of diameter $10-30 \mathrm{~km}$ have radiated away their radioactive heat fast enough not to have become more than partially melted near their centres?

I have difficulty in believing that a complete hierarchy of asteroids ranging in size down to meters in diameter was not formed originally. Below a certain limit of size some elimination must have occurred as a result of the loss of solar-nebula gas. But at diameters of the order of $3 \mathrm{~km}$, some 55000 now exist (Baade, 1934; Kuiper et al., 1958). Are they all broken fragments of larger asteroids, or do they include a good fraction that were never much larger? The present particulate erosion rate at the very high value of $1 \mu / \mathrm{yr}$ (Whipple, 1967) would amount to a maximum diameter loss of $10 \mathrm{~km}$ in $5 \times 10^{9} \mathrm{yr}$. A much smaller erosional loss than this value, which is appropriate to cometary meteoroids, would be more reasonable for 'half-baked' asteroidal material. Thus, rather small asteroids might have persisted. The collisional and erosional destruction rates for asteroidal material are, however, unknown over the life of the solar system, particularly during the early period.

The surprisingly large fraction of Mars-crossing asteroids belonging to families suggests that collisional break-up is rather common in this part of the solar system and that the very small bodies of weak construction may mostly have been eliminated by now. On the other hand, Anders (1965) shows evidence that the process of collisional break-up is not far advanced. But how far has it advanced for weak asteroidal structures of original diameters less than $30 \mathrm{~km}$ ?

Several questions of lunar-crater formation and rates are also related to these problems of asteroidal and cometary objects in Earth-crossing orbits.

This note is intended not to answer questions but to present more possibilities and pose more questions in the hope that investigators will search for clues, both theoretical and observational, that may bear on these problems. Particularly I hope that cometary nuclei or cometary fragments will not always be credited with producing observed phenomena that involve bodies of weak physical structure - at least unless the true nature of these friable bodies or their orbits has been demonstrated.

\section{References}

Anders, E. (1965) Icarus, 4, 399.

Baade, W. (1934) Publ. astr. Soc. Pacific, 46, 54.

Fesenkov, V.G. (1961) Astr. Zu., 38, 577 (also in 1962, Soviet Astr., 5, 441).

Kuiper, G.P., Fujita, Y., Gehrels, T., Groeneveld, I., Kent, J., Van Biesbroeck, G., Van Houten, C.J. (1958) Astrophys. J. Suppl. Ser., 3, 289.

Öpik, E.J. (1966) Univ. Liège Inst. Astrophys. Coll. $8^{\circ}, 21,575$.

Whipple, F.L. (1967) Int. Symp. on the Zodiacal Light and the Interplanetary Medium, Honolulu (to be published). 


\section{DISCUSSION}

Dohnanyi: Since you have considered the distribution of particles up to a distance of $3.5 \mathrm{AU}$, I wonder if you have also considered the influence of large asteroids in the asteroidal belt?

Whipple: Perhaps 3.5 AU is too small a cut-off but it is near the outer edge of the asteroid belt and near the region of serious Jupiter perturbations. Perhaps 4.5 AU would be better. Dr. Dohnanyi and $I$ found in Hawaii that we were attacking the larger-body problem almost identically. I am glad he has had the time to carry out the investigation, which he will present next.

Fedynskij: What do you think about the probable explosion of a major planet 50 million years ago, as an explanation of the exposure age of the stony meteorites?

Whipple: A planetary explosion $50 \times 10^{6}$ years ago does not fit the stone exposure age distribution without erosion. Also there is some evidence for an iron asteroid break-up some $600 \times 10^{6}$ years ago. Stony break-ups would surely be much more frequent. 